\title{
Terapia Ocupacional, discapacidad y género: la interseccionalidad como apertura hacia reflexiones pendientes
}

\author{
Occupational therapy, disability and gender: \\ intersectionality as an opening to pending reflections
}

\author{
Débora Ester Grandón Valenzuela' \\ Recibido: 16 de agosto 2017 - Enviado para modificación: 10 de noviembre 2017 - Aceptado: 8 de diciembre 2017 \\ Grandón, D.E. (2017). Terapia Ocupacional, discapacidad y género: la interseccionalidad como apertura hacia \\ reflexiones pendientes. Revista Ocupación Humana, 17(2), 34-45.
}

\begin{abstract}
RESUMEN
De forma tácita, la interrogante que guía el presente trabajo reflexivo es la pregunta por las ausencias dentro de la producción del conocimiento en Terapia Ocupacional. Particularmente, el cuestionamiento por la ausencia de las perspectivas de género y feministas en la comprensión de la discapacidad conduce hacia un análisis desde la interseccionalidad, tensionando al Modelo Social de Discapacidad como último modelo teórico, de manera que nos permita una mayor complejidad para comprender dicho fenómeno social. El examen que aquí se realiza busca apelar al permanente ejercicio reflexivo del gremio, considerando que lo que seguimos transmitiendo académicamente representa hegemonías del saber que debemos cuestionar, para así dar paso a nuevos saberes, a la emergencia de reflexiones que surjan desde la vida cotidiana y, de esta manera, constituirnos en actores políticos con un posicionamiento que vaya de la reflexión a la acción, y hacia la reflexión otra vez.
\end{abstract}

\section{PALABRAS CLAVE}

Terapia ocupacional, género, persona con discapacidad, formación profesional, derechos humanos

\begin{abstract}
The interrogation that tacitly leads this reflexive work is the question about the absences in the production of knowledge from occupational therapy. Specifically, the questioning for the absence of gender and feminist perspectives on the comprehension of disability leads to an analysis from intersectionality, putting a pressure on the social model of disability as the ultimate theoretical model that allows for a larger complexity to understand the social phenomenon of disability. The analysis herein seeks to appeal to a permanent reflexive exercise of occupational therapists, considering that what we are transmitting in the academia
\end{abstract}

${ }^{1}$ Terapeuta Ocupacional. Maestranda en Estudios de Género y Cultura, Mención Ciencias Sociales, Universidad de Chile. Docente adjunta, Escuela de Terapia Ocupacional, Universidad Autónoma de Chile. Docente adjunta, Escuela de Terapia Ocupacional, Universidad Andrés Bello. Santiago de Chile. dlunagrandon@gmail.com 
represents predominant knowledge that we must question, so we can open up to knowledge, to the emergence of critical thoughts derived from everyday life, so we can become political actors, with a position that goes from reflection to action, and back again to reflection.

\section{KEY WORDS}

Occupational therapy, gender, disabled persons, professional training, human rights

\section{Introducción}

"Que mucho de lo que no existe en nuestra sociedad es producido activamente como no existente, y por eso la trampa mayor para nosotros es reducir la realidad a lo que existe"

(De Sousa Santos, 2006, p. 23).

Tendemos a pensar, quizá por la forma en que nos han enseñado a pensar el mundo, que solo aquello evidente, eso que compone las historias oficiales en los distintos saberes, compone lo verdadero. Sin embargo, obviamos el hecho de que aquello comprendido como verdad -o presencia-, ha sido puesto en ese lugar y repetido incesablemente por generaciones, hasta ocupar el estatus de verdad. Pero ¿cuál es el lugar de todas esas cosas que no logran posicionarse como presencias dentro de los relatos y saberes? ¿Qué tienen que decir todos esos saberes, que más que olvidados, pareciesen estar negados? ¿Qué tienen que decir esas ausencias, que aunque invisibles, existen en su condición de inexistentes?

Pareciera ser que la discapacidad constituye una de esas ausencias históricas que a través de distintos movimientos, apariciones políticas y sensibilizaciones busca constantemente salir del espacio de lo ausente para hacerse una presencia. Y efectivamente, en muchos campos lo ha logrado. Solo a modo de referencia histórica, he de mencionar la Convención Internacional de Derechos de las Personas con Discapacidad (ONU, 2006), que al abrir su suscripción en 2007 fue firmada por 88 países y 44 incurrieron como signatarios en su protocolo facultativo (Naciones Unidas, Enable, s.f.). Sin duda este hecho constituye un hito y un amparo legal para las personas en situación de discapacidad, pese a que podamos vislumbrar discordancias entre las políticas y praxis de los países signatarios.

Desde la formación académica en Terapia Ocupacional, al contrario de la sociedad civil general, la discapacidad constituye una temática abordada y discutida de forma permanente, por lo que muchas veces, desde los dispositivos de salud especialmente, se considera a las y los terapeutas ocupacionales como "expertos en discapacidad". Sin embargo, las reflexiones sobre el tema suelen terminar con el Modelo Social, considerando este como el último y más complejo punto de discusión, y por tanto, de transmisión académica.

Desde el Modelo Social se ha entendido la discapacidad, no como un atributo individual, sino como un conjunto de condiciones que, al darse juntas, 
producen exclusión social para algunas/os sujetos al no garantizar su accesibilidad física o ciudadana. Si bien el modelo ha constituido un cambio paradigmático fundamental en la academia -desplazando perspectivas biomédicas en las que se consideraba la discapacidad como una enfermedad individual, o rehabilitadoras, que en la misma lógica buscaban normalizar a sujetos "enfermos" para su integración a la sociedad-, la visión de la discapacidad como un todo homogéneo, como un fenómeno uniforme, esconde exclusiones que necesitan ser visibilizadas para poder reflexionar sobre particularidades que hoy precisan nuevas miradas.

En este sentido, pensar y reflexionar la vivencia social de la discapacidad desde un enfoque de género puede favorecer la emergencia de problemáticas que han quedado ocultas tras el relato unificador del Modelo Social. Sin la intención de desechar este modelo, me propongo más bien enriquecerlo desde el análisis de la relación de poder que más tempranamente introyectamos: el género.

\section{Sobre el Modelo Social de Discapacidad}

Como ya se adelantó, el Modelo Social de Discapacidad ha constituido un hito a nivel paradigmático, complejizando el entendimiento que se había dado a la discapacidad. Agustina Palacios (2008) señala que este modelo surgió a fines de los años sesenta, principalmente en Europa y Estados Unidos. En este último país se dio paralelamente o apoyado por otras demandas, como aquellas en contra de opresiones por raza y clase; sin embargo, la discapacidad permaneció como un fenómeno ligado a lo individual y médico. Pese a la dificultad por posicionar sus denuncias y demandas en la palestra política, personas en situación de discapacidad constituyeron el Movimiento de Vida Independiente, el cual logró, por ejemplo, la desmedicalización y desinstitucionalización de sus condiciones de vida.

Un hito importante para la constitución de dicho movimiento fue el ingreso de Ed Roberts a la Universidad de California en Estados Unidos; quien según señala Palacios (2008), pese a haber sido considerado en su momento como "discapacitado severo", logró ingresar a estudiar Ciencias Políticas desafiando el entorno no accesible del establecimiento universitario. Otras personas en situación de discapacidad tomaron su ejemplo y comenzaron a ocupar ese espacio que históricamente se les había negado, generando discusiones políticas sobre las estructuras de la universidad. En sus tiempos de estudiante, Roberts se dedicó a estudiar el feminismo, pues consideraba que existían muchas analogías entre las situaciones vividas por las mujeres y las de las personas que se enfrentaban a la discapacidad, realizando un re-lectura de la consigna feminista: "la anatomía no es destino". Así, Roberts comparó que tanto las mujeres como las personas en situación de discapacidad buscaban tener control sobre sus propios cuerpos y decisiones, cuestionando, en su caso, la medicalización de su vivir.

Es interesante señalar que ya para los años sesenta Roberts advertía ese paralelismo entre feminismo y discapacidad, notando una evidente relación entre ambos; sin embargo, esta interseccionalidad continúa siendo es- 
casamente abordada por los estudios de género y por los que realiza Terapia Ocupacional sobre discapacidad.

Sin profundizar más en los hitos que fueron consolidando la comprensión social y política de la discapacidad, que dio paso al Modelo Social de la Discapacidad, diré que tal modelo dejó de explicar este fenómeno como un atributo de las personas, para comprenderlo como el resultado de barreras diversas que coartan el acceso y la participación para algunas personas. Desde este modelo se comprende que "las personas con discapacidad son discapacitadas como consecuencia de la negación por parte de la sociedad de acomodar las necesidades individuales y colectivas dentro de la actividad general que supone la vida económica, social y cultural" (Palacios, 2008, p.121). Así, el énfasis al abordar la discapacidad pasa de lo médico individual, y por tanto de la necesidad de "rehabilitar", hacia su visibilización como una consecuencia de la opresión ejercida por sociedades construidas de formas que no consideran la diversidad ni la accesibilidad universal.

Este modelo logró posicionarse como paradigma contemporáneo para interpretar la discapacidad, y desde él se sustenta la Convención Internacional sobre los Derechos de las Personas con Discapacidad (ONU, 2006), situándola ya no como un tema atinente a los sectores de salud, sino como un asunto social y de derechos humanos. Esto cambia radicalmente la forma de analizar y concebir la discapacidad: el terreno de lo social permite situar el debate no en sujetos individuales con un padecimiento, sino en cómo existen determinadas formas de construir esce- narios sociales en donde la divergencia y la diferencia humana son negadas; no todas las personas pueden acceder a un pleno ejercicio de derechos y ocupaciones, por barreras que van desde lo arquitectónico, lo comunicacional, lo social y lo actitudinal hasta lo legislativo, por mencionar algunas.

Otro interesante aporte de la Convención (ONU, 2006) es que releva la importancia de incluir la perspectiva de género al momento de fomentar el ejercicio pleno de los derechos de las personas en situación de discapacidad, reconociendo que a partir del género pueden darse formas particulares de abuso, violencia y explotación. Sin embargo, ¿hemos sido capaces de profundizar la compleja relación que se teje entre el género y la discapacidad desde nuestros saberes académicos y profesionales? ¿Hemos logrado profundizar en esta primaria relación de poder, que es el género, y en cómo permea las relaciones sociales y las ocupaciones? El género, como lente analítico para observar la realidad, nos mostrará situaciones que han sido históricamente producidas como ausencias, desde la construcción hegemónica y masculina de las ciencias y saberes, y que hoy es necesario hacer visibles.

\section{Interseccionalidad entre género y discapacidad}

Desde que somos infantes, una de las cosas que más rápido se nos enseña es qué somos: niño o niña, hombre o mujer, considerando que esta distinción binaria es fundamental y necesaria para la construcción de identidad. Se nos viste de colores que afirmen dicha identidad, se nos enseñan labores, 
juegos y juguetes apropiados, se nos ensalzan habilidades o actitudes consideradas deseables para el género que socialmente se nos asigna. Desde nuestra más primaria constitución como seres sociales se nos enseña a representar roles y funciones propias para el género que, en base a nuestro sexo biológico, se nos enseña a ser.

Esta construcción social y simbólica de los géneros binarios hombre/mujer lleva en sí desigualdades que actúan como una relación de poder. Joan Scott (1990) introduce a los estudios de género la comprensión de la categoría género como una forma primaria de relaciones de poder, señalando que es un elemento que constituye las relaciones sociales a partir de las diferencias que existen entre los sexos. Así, la categorización del género con base en la diferencia sexual de los cuerpos se traduce en relaciones sociales desiguales, en donde la mujer ha quedado sometida y subordinada; mecanismo de poder en camuflaje, pues al basarse en algo "natural", como la diferencia de los cuerpos biológicos, hace que la desigualdad construida como resultado de esta también sea concebida como natural y, por tanto, sea naturalizada e invisibilizada.

La construcción del género femenino, entendida entonces como el polo subyugado dentro de una relación de poder, se ha expresado en diversas formas que no han sido ejercidas sólamente desde los hombres o las instituciones hacia las mujeres, sino que además han sido introyectadas por las mismas mujeres en su proceso de subjetivación, complejizando el panorama. Marcela Lagarde (2005) señala que existen estereotipos de construcción de lo femenino instaurados como cautiverios, en los cuales lo común ha sido constituirse como un ser-de-los-otros y un ser-paraotros. Es interesante visibilizar que la estrategia del cautiverio no reside fuera de las mujeres, sino en ellas mismas, en sus afectos y en su conciencia, tal y como señala Lagarde (2005) al decir que:

el principal carcelero de las mujeres son sus necesidades y su conciencia, es decir, su subjetividad formada, apoyada y reproducida por el conjunto de relaciones e instituciones económicas, sociales, jurídicas, religiosas, eróticas y políticas, que hacen a las mujeres cumplir un orden social convertido en orden vital cósmico. (p.165)

¿Cuál es el orden vital cósmico del que habla Lagarde? ¿Se instituye este por igual en toda mujer? ¿Puede siquiera hablarse de "la" mujer como un universal? Todo el proceso de socialización para convertir a las mujeres en mujeres, siguiendo a De Beauvoir (2013) al entender que ser mujer es haber sido devenida como tal, es un proceso multidimensional complejo que abarca, desde las instituciones como la familia, la escuela y el Estado, hasta las relaciones sociales más cotidianas, en donde se reproduce constantemente el lugar, rol y estereotipo que la mujer ocupa en la cultura patriarcal, particularmente como madresposas. Este proceso, esta forma de ser socializadas, se va introyectando psíquicamente en las mujeres hasta formar parte de su conciencia y de lo más íntimo de su ser. Así se sostiene el cautiverio de las mujeres, presas y oprimidas desde el mismo proceso de subjetivación, hecho que explica la complejidad de la deconstrucción de los estereotipos patriarcales que ellas y las estructuras sociales 
reproducen día a día. Lagarde (2005) señala también que el hecho de que las mujeres estén cautivas radica en que han sido privadas de su autonomía, de su independencia, del propio gobierno sobre sí mismas, de la posibilidad de escoger y de tomar decisiones sobre los hechos más fundamentales de sus vidas y del mundo.

Es indispensable considerar que el género no existe de manera aislada, siempre se da en relación, y que más que una categoría analítica es una relación encarnada desde diversos lugares sociales y condiciones socio-históricas que impiden que sea, en sí misma, una estructura homogénea y universal. Desde el feminismo se ha comprendido que el género coexiste con otras estructuras que lo atraviesan, complejizan y diferencian. Así, se ha concebido la interseccionalidad en referencia a esos múltiples entrecruzamientos que el género sostiene desde la vida cotidiana con otras "categorías", lo que inmediatamente tensiona la noción de "la" mujer como un universal y permite ver la multiplicidad de identidades fragmentadas que componen la vivencia de la feminidad. Así, "las mujeres comparten como género la misma condición genérica, pero difieren en cuanto a sus situaciones de vida y en los grados y niveles de opresión" (Lagarde, 2005, p.79). La interseccionalidad nos permite visibilizar que no es lo mismo ser una mujer blanca, profesional, heterosexual, que vive en una metrópoli, que una mujer indígena que vive en una zona rural y encarna alguna discapacidad.

Debe tenerse en cuenta que la interseccionalidad no implica la mera adición de variables cual operación aritmética, sino que permite pensar "contextos de dominación construidos históricamente" (Viveros, 2016, p.8). Ello permite deconstruir la imagen de la mujer como un universal y, por tanto, sujeta siempre a las mismas vivencias y problemáticas ligadas solo al género. Podría decirse que la interseccionalidad es una herramienta analítica que permite visibilizar la diferenciación de lugares sociales que se crean a partir de las relaciones de poder que cruzan las estructuras sociales. Así, es fundamental visibilizar cómo existe un tejido entre las relaciones de género y otras relaciones sociales, el cual crea mujeres distintas.

Para el caso del presente análisis, pensar el entrecruce entre género y discapacidad permite visibilizar posiciones y vivencias particulares. Por una parte, las mujeres en situación de discapacidad no siempre son concebidas y reconocidas como mujeres, de hecho, su particularidad ha estado históricamente ausente en los mismos movimientos feministas, los cuales sí han incorporado luchas diferenciales como las de las feministas indígenas o las feministas negras (black feminism). Por otra parte, las mujeres en situación de discapacidad tampoco cumplen el estándar estético y funcional impuesto por los cánones de normalidad, siendo marginadas y discriminadas de diversos espacios sociales. Esto nos muestra la existencia de, al menos, dos luchas para las mujeres en situación de discapacidad: una, por la inclusión social y ciudadana, y otra, contra las opresiones propias que las relaciones de género tienen sobre sí, en una expresión diferencial. Es importante reconocer que no debemos asumir a priori que una mujer en situación de discapacidad estará oprimida solo por encarnar estas 
categorías y, por tanto, verla desde un enfoque victimista, sino desde uno de derechos humanos que permita visibilizar sus resistencias y formas políticas.

Más allá de limitarse a pensar que las mujeres en situación de discapacidad viven una doble opresión (por género y discapacidad), la interseccionalidad nos permite pensar cómo la clase social o la raza ${ }^{2}$ también configuran otras formas de encarnar sujetos sociales. Me he enfocado en el cruce del género con la construcción social de la discapacidad para enfatizar cómo el modelo que más seguimos transmitiendo académicamente en Terapia Ocupacional no nos permite visibilizar, de manera suficiente, estas identidades fragmentadas que el lente analítico interseccional nos revela.

El entretejido diferencial entre estas posiciones mujer/discapacidad ha sido motivo de reflexiones más bien recientes, que poco a poco han permitido que esta histórica ausencia, tanto en los feminismos como en los estudios específicos sobre discapacidad, y en la misma Terapia Ocupacional, emerja como presencia. En este sentido, resulta interesante señalar que han surgido a lo largo del mundo feministas en situación de discapacidad que tienen puntos de reivindicación comunes con el feminismo tradicional, como el ejercicio de la sexualidad, la maternidad y las decisiones acerca de su propio cuerpo (Serra, 2014). Sin embargo, la manera de abordar estas demandas "comunes" es radicalmente distinta.
A diferencia de las luchas feministas tradicionales, las feministas en situación de discapacidad han reclamado su derecho a ejercer los roles tradicionales que el imaginario social del ser-mujer conlleva, como el de ser madres, puesto que ellas "han visto vulnerados sus derechos sexuales y reproductivos a través de abortos y esterilizaciones forzosas, de la privación del acceso a una maternidad informada o de la negación de su sexualidad e intimidad" (Pérez de la Merced, 2017, p.155). Mientras que feminismos mayoritarios luchan por el derecho al aborto y a una maternidad libre, no impuesta, las mujeres en situación de discapacidad buscan el derecho de ejercer la maternidad. Son varias las investigaciones (Cruz, 2004; Serra, 2014; Pérez de la Merced, 2017) que hacen hincapié en que las mujeres en situación de discapacidad experimentan mayores "limitaciones en el acceso a información, en la asistencia sanitaria $y$, por ende, en dificultades para decidir cuestiones relacionadas con el cuidado de su cuerpo: su salud, el ejercicio de su sexualidad y la decisión de tener o no hijos" (Cruz, 2004, p.226), lo que evidencia la potente restricción social que tienen en la vida cotidiana, particularmente en lo relativo a su sexualidad.

Retomando las líneas que propone María Laura Serra sobre las demandas específicas de mujeres feministas en situación de discapacidad (ejercicio de la sexualidad, la decisión acerca de su propio cuerpo y el ejercicio de la maternidad), luego de lo investigado y en mi consideración personal, esbozo algunas

\footnotetext{
${ }^{2}$ Utilizo el concepto de raza en forma crítica, reivindicativa, puesto que en ningún caso considero que efectivamente existan razas de personas. La utilización del concepto es en sí una estrategia para visibilizar que existe una estructura social creada para distinguir y discriminar, que ha sido la raza.
} 
de las exclusiones específicas que ellas viven, especialmente en lo que se refiere a sus derechos sexuales y reproductivos.

\section{El cuerpo asexuado}

El cuerpo de las mujeres en situación de discapacidad se considera estéticamente anormal, puesto que no cumple con los elevados y exigentes estándares de los estereotipos del cuerpo femenino. Los cuerpos de las mujeres con discapacidad se conciben como lo indeseable, lo anómalo, y al no cumplir el patrón socialmente transmitido -tremendamente cosificante y patriarcal-, se consideran asexuados en doble vía, en tanto carentes de deseo sexual, por una parte, y ausentes de encarnar cuerpos que resulten deseables, por otra. Así, mujeres que viven situaciones de discapacidad son simbólica y fácticamente apartadas del escenario sexual, constituyendo al ejercicio de la sexualidad como un fuerte tabú.

De todos modos, no deben obviarse las cifras que señalan que las mujeres en situación de discapacidad-especialmente intelectual-, son más vulnerables a abusos sexuales por parte de sus familiares o, incluso, de asistentes y profesionales de la salud. Lo anterior tensiona que, pese a ser un cuerpo socialmente visto como asexuado, puede utilizarse como medio para la satisfacción sexual de otro, que violentamente abusa de su posición y poder (IMSERSO, Universidad Autónoma de Barcelona - Asociació Dones No Estàndars, 2002).

\section{Maternidad negada}

A las mujeres consideradas discapacitadas se les aleja del rol de madre, no meramente como un acto pasivo, puesto que incluso se les somete a la esterilización involuntaria y forzada; como ocurre a algunas mujeres con síndrome de Down. Desde el imaginario, la reproductividad de una mujer no estándar debe limitarse, coartarse, por lo que la maternidad, que a las mujeres no discapacitadas se transmite como un destino vital cósmico, les es negada a aquellas en situación de discapacidad a través de distintos tipos de barreras. A estas mujeres se les excluye, en primer lugar, de la decisión, y luego, de la posibilidad de participar en el rol de ser madres; lo que da cuenta de que, entre líneas, ser madre no es para todas las mujeres por igual, solo para aquellas que encarnan la normalidad femenina.

\section{Desinformación sobre sexualidad}

Considerando el amplio espectro que puede abarcar la noción de discapacidad, muchas veces la información sobre sexualidad, relaciones sexuales y métodos anticonceptivos no es puesta en términos que resulten accesibles para todas las mujeres. Esta accesibilidad no abarca solo lo sensorial o lo cognitivo, sino incluso lo actitudinal; si un profesional de salud, desde su propia construcción simbólica del mundo, sus prejuicios y valores, no concibe a una mujer en situación de discapacidad como ser sexuado, probablemente omitirá orientarla en torno al tema, pues considera innecesario que maneje esa información, asumiendo tácitamente que no es partícipe de la vida y el goce sexual.

\section{Discusión}

La desinformación sobre la propia sexualidad, la negación de la maternidad y la visión de las mujeres en situación 
de discapacidad como seres asexuados nos brindan ejemplos concretos de exclusiones que se gestan desde el cruce entre género y discapacidad, y que permanecen tremendamente invisibilizadas, aún desde el Modelo Social de Discapacidad. Desde la lectura de la discapacidad como homogeneidad, en donde se concibe que hombres y mujeres tienen demandas iguales por la inclusión educativa, laboral o social, las especificidades derivadas de la construcción socio-simbólica del género permanecerán como una ausencia.

El análisis interseccional entre discapacidad y género permite visibilizar exclusiones específicas a las que se enfrentan un grupo de mujeres. En primer lugar, son excluidas de la construcción socio-simbólica del ser mujer, siendo consideradas como "menos mujeres" o incluso como "niñas", lo que les impide participar de aquellos espacios construidos como lo femenino. Es fundamental señalar que, pese a que dicha construcción es en sí misma patriarcal y opresiva, al excluir a las mujeres en situación de discapacidad de estos espacios se les priva de la identificación social como mujeres. En segundo lugar, son excluidas en términos de su accesibilidad al mundo social, laboral y político, espacios que cuando logran penetrar, les son "reconocidos" bajo el asistencialismo, la caridad y, muchas veces, sujetas a diversas inequidades.

En este sentido, la interseccionalidad permite develar ordenamientos políticos y culturales que se inscriben en la base de la manera en que organizamos el mundo, por lo que debe comprenderse no solo como una herramienta analítica, sino como una episte- mología de resistencia que ayuda a

...comprender el orden socio-cultural del poder, a develar la forma como el poder construye subjetividades a través de la (re)composición por sexo/género, etnicidad/raza, heterosexualidad/homosexualidad, o capacidad/discapacidad, y a pensar en la producción de subjetividades alienadas para acentuar los procesos de interacción entre subjetividades y cuerpos. (Múnevar, 2012, p.58).

Así, la interseccionalidad visibiliza, mediante lo que Dora Múnevar (2012) reconoce como marcadores de diferencia, subjetividades emergentes, subjetividades otras que encarnan vivencias múltiples e interconectadas entre sí, las cuales se expresan en la cotidianidad. Los cuerpos, como producto social, inscriben en sí lo político, encarnando las distintas estructuras y construcciones socio-culturales que producen el poder, el privilegio y la marginación (Múnevar, 2012), por lo que iluminar los marcadores de diferencia, en sus múltiples entrecruzamientos, permite la emergencia de vivencias que han quedado borradas por la supremacía de las ciencias.

Lo anterior nos lleva a re-pensar nuestro rol en la transmisión académica, puesto que incluir el pensamiento interseccional y las perspectivas feministas en nuestro ejercicio teórico, más que una "actualización técnica", constituye un ejercicio político, al considerar que:

La academia sería entonces una de las instituciones encargadas de gestionar la opresión derivada del dominio estructural mediante la cual se valida socialmente la opresión. Esto 
sucedería a partir de la generación de un conocimiento particular que silencia y pone acentos sobre determinados discursos. La academia, como dominio hegemónico, contribuiría a reproducir las estructuras de poder en la intersubjetividad individual y colectiva... (Cubillos, 2015, p.128)

Las presentes reflexiones no son cómodas, pues conllevan una responsabilidad ética y política que nos mueve a sacudir el polvo de aquello que hemos producido como inexistente (De Sousa, 2006), buscando allí saberes que han quedado silenciados por las estructuras hegemónicas del poder.

\section{Conclusiones}

El Modelo Social, sin duda, ha contribuido a un fuerte cambio paradigmático, desplazando la comprensión biomédica, reduccionista e individual de la discapacidad hacia un entendimiento de esta como interrelación de factores sociales que incluyen los de tipo económico, cultural, actitudinal, arquitectónicos, de acceso, políticos, etc. Así, discapacidad es el resultado de condiciones sociales que impiden el libre y autónomo ejercicio ocupacional de algunos/as sujetos.

Sin embargo, desde la academia se ha tendido a considerar este nivel de análisis como el último, deteniendo, en alguna medida, nuevas comprensiones emergentes sobre el complejo fenómeno social de discapacidad. Se hace necesario, en primer lugar, deshomogeneizarla, para poder entrever en ella particularidades y fragmentaciones que hasta ahora han permane- cido ausentes, como el entrecruce género - discapacidad.

Esta interseccionalidad, como clave analítica y ejercicio epistemológico, permite visibilizar y pensar desde las particularidades que se gestan como producto de dos categorías encarnadas, o marcadores de diferencia, considerando que cada uno de ellos, por sí mismo, ha implicado discriminación y exclusión. Por tanto, la interseccionalidad da cuenta de las particularidades de subjetividades emergentes que no pueden estandarizarse bajo el alero de la discapacidad como un totalizante.

Este artículo pretende instar hacia un ejercicio reflexivo de los y las terapeutas ocupacionales, quienes considerándonos como actores sociales y políticos, poseemos una responsabilidad ética de estar permanentemente examinando la realidad social contemporánea, para así ser capaces de promover cambios estructurales que permitan el ejercicio libre de ocupaciones dignas, significativas y realizantes para todos y todas.

Por todo lo anterior, este artículo busca llamarnos al no-conformismo, a no dar nuestras reflexiones por acabadas, a cuestionarnos aquello que concebimos como saber. Nos insta a desarrollar epistemologías críticas y de resistencia a aquello que sostenemos como conocimiento, buscando no solo en lo evidente, en lo existente, sino examinando también aquellas cosas que han sido producidas como ausencias en la historia.

Abrirnos hacia un enfoque de género, no solo de la discapacidad sino del mismo ejercicio profesional y de las 
ocupaciones, nos permite acercarnos cada vez más a reflexiones desde la diferencia, a la construcción de una multiplicidad de terapias ocupacionales ${ }^{3}$ que no extingan su saber, sino que, sujetas a la participación política de lo cotidiano, reconozcan las múltiples formas de opresión que vivimos y permitan gestar, desde lo micropolítico, transformaciones sociales profundas que alegren y emancipen nuestro vivir.

\section{Referencias}

Cubillos, J. (2015). La importancia de la interseccionalidad para la investigación feminista. Oxímora Revista Internacional de Ética y Política, (7), 119-137.

Cruz, M.P. (2004). Mujeres con discapacidad y su derecho a la sexualidad. Política y Cultura, 22, 147-160.

De Beauvoir, S. (2013) El segundo sexo. Buenos Aires: Debolsillo.

De Sousa Santos, B. (2006). Renovar la teoría crítica y reinventar la emancipación social (encuentros en Buenos Aires). Buenos Aires: CLACSO.. Disponible en http:// biblioteca.clacso.edu.ar/clacso/coediciones/20100825032342/critica.pdf

Iglesias, M. (2003). Mujeres y Discapacidad: la doble discriminación. Revista Emakunde, (50), 6-11. Disponible en http://www.emakunde.euskadi.eus/ contenidos/informacion/sen_revista/es_ emakunde/adjuntos/revista.emakunde.50.pdf

IMSERSO, Universidad Autónoma de Bar- celona - Asociació Dones No Estàndars. (2002). Indicadores de exclusión social de mujer y discapacidad. Disponible en http://www.guiaviolenciadegenero.com/pdf/indicadores_exclusion_mujer_discap.pdf

Lagarde, M. (2005). Cautiverios de las mujeres: madresposas, monjas, putas, presas y locas. México: Universidad Nacional Autónoma de México.

Múnevar, D. (2012) Interseccionalidad y otras nociones. En M. Zapata, S.García, J. Chan de Avila (eds.) La interseccionalidad en debate (pp.56-65). Berlín: Instituto de Estudios Latinoamericanos de la Freie Universität Berlin.

Naciones Unidas, Enable. (s.f.). Los derechos y la dignidad de las personas con discapacidad. New York: ONU. Disponible en: http://www.un.org/spanish/ disabilities/default.asp?id=497

ONU. (2006). Convención Internacional sobre los Derechos de las Personas con Discapacidad. Disponible en http:// www.un.org/disabilities/documents/ convention/convoptprot-s.pdf

Palacios, A. (2008). El Modelo Social de Discapacidad: orígenes, caracterización y plasmación en la Convención Internacional sobre los Derechos de las Personas con Discapacidad. Madrid: Grupo Editorial CINCA.

Pérez de la Merced, H. (2017). Ciudadanas en la encrucijada: interseccionalidad y ciudadanía íntima de las mujeres con diversidad funcional. Investigaciones Feministas, 8(1), 151-163.

\footnotetext{
${ }^{3}$ Retomo esta acertada noción que mi querido profesor Alejandro Guajardo Córdoba ha utilizado en diversos escritos de su autoría.
} 
Scott, J. (1990). El género: una categoría útil para el análisis histórico. En J. Amelang y M. Nash (eds.), Historia y Género: las mujeres en la Europa moderna y contemporánea (pp.23-56). Valencia: Alfons el Magnanim.

Serra, M. (2014). Feminismo y Discapacidad. Derechos y Libertades, época II (31), 251-272. Disponible en https://e-archivo.uc3m.es/bitstream/handle/10016/22527/DyL-2014-31-serra.pd$\mathrm{f}$ ? sequence $=1$
Viveros, M. (2016). La interseccionalidad: Una aproximación situada a la dominación. Debate Feminista, 52 (octubre), 1-17. Disponible en http://www. sciencedirect.com/science/article/pii/ S0188947816300603 